\section{Michigan Technological 1 8 85 University}

Michigan Technological University Digital Commons @ Michigan Tech

7-2007

\title{
Room temperature operational single electron transistor fabricated by focused ion beam deposition
}

P. Santosh Kumar Karre

Michigan Technological University

Paul Bergstrom

Michigan Technological University

Follow this and additional works at: https://digitalcommons.mtu.edu/ece_fp

Part of the Electrical and Computer Engineering Commons

\section{Recommended Citation}

Karre, P. S., \& Bergstrom, P. (2007). Room temperature operational single electron transistor fabricated by focused ion beam deposition. Journal of Applied Physics, 102(2). http://dx.doi.org/10.1063/1.2761837

Retrieved from: https://digitalcommons.mtu.edu/ece_fp/1

Follow this and additional works at: https://digitalcommons.mtu.edu/ece_fp

Part of the Electrical and Computer Engineering Commons 


\section{Room temperature operational single electron transistor fabricated by focused ion beam deposition}

P. Santosh Kumar Karre, Paul L. Bergstrom, Govind Mallick, and Shashi P. Karna

Citation: Journal of Applied Physics 102, 024316 (2007);

View online: https://doi.org/10.1063/1.2761837

View Table of Contents: http://aip.scitation.org/toc/jap/102/2

Published by the American Institute of Physics

\section{Articles you may be interested in}

Silicon single-electron quantum-dot transistor switch operating at room temperature Applied Physics Letters 72, 1205 (1998); 10.1063/1.121014

Metal-nanoparticle single-electron transistors fabricated using electromigration Applied Physics Letters 84, 3154 (2004); 10.1063/1.1695203

Single-electron transistors operating at room temperature, fabricated utilizing nanocrystals created by focusedion beam

Applied Physics Letters 80, 2168 (2002); 10.1063/1.1458685

Room-temperature Al single-electron transistor made by electron-beam lithography

Applied Physics Letters 76, 2256 (2000); 10.1063/1.126313

Single-electron transistors in heterostructure nanowires

Applied Physics Letters 83, 2052 (2003); 10.1063/1.1606889

Complementary digital logic based on the "Coulomb blockade" Journal of Applied Physics 72, 4399 (1998); 10.1063/1.352206

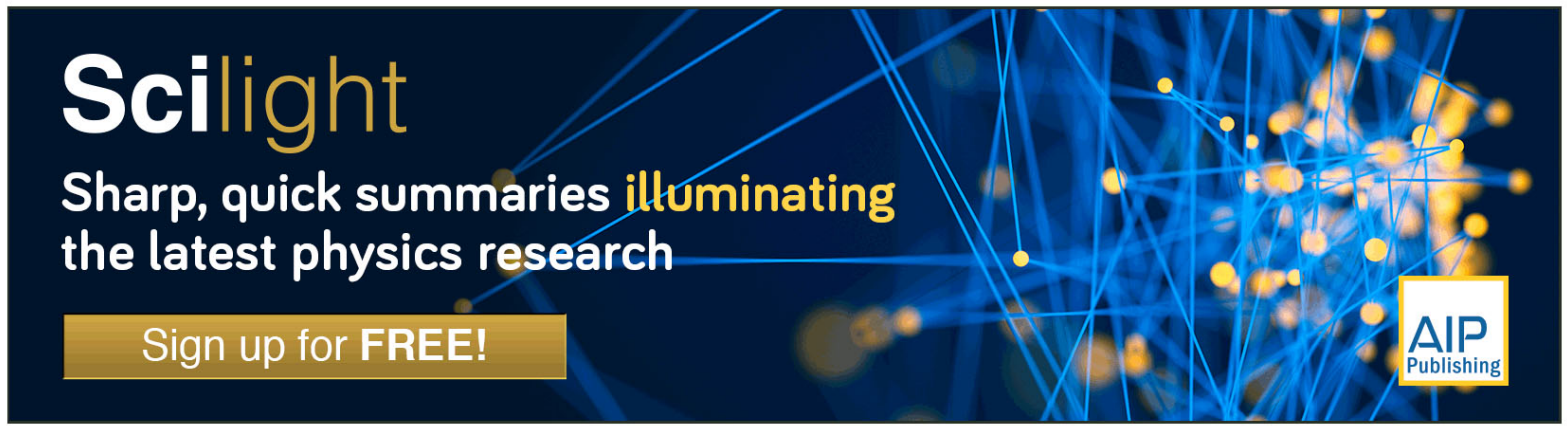




\title{
Room temperature operational single electron transistor fabricated by focused ion beam deposition
}

\author{
P. Santosh Kumar Karre ${ }^{\text {a) }}$ and Paul L. Bergstrom \\ Multi Scale Technologies Institute, Michigan Technological University, Houghton, Michigan 49931 \\ and Department of Electrical and Computer Engineering, Michigan Technological University, \\ Houghton, Michigan 49931 \\ Govind Mallick and Shashi P. Karna \\ Weapons and Materials Research Directorate, US Army Research Laboratory, Building 4600, \\ Aberdeen Proving Ground, Maryland 21005-5069
}

(Received 6 November 2006; accepted 14 June 2007; published online 27 July 2007)

\begin{abstract}
We present the fabrication and room temperature operation of single electron transistors using $8 \mathrm{~nm}$ tungsten islands deposited by focused ion beam deposition technique. The tunnel junctions are fabricated using oxidation of tungsten in peracetic acid. Clear Coulomb oscillations, showing charging and discharging of the nanoislands, are seen at room temperature. The device consists of an array of tunnel junctions; the tunnel resistance of individual tunnel junction of the device is calculated to be as high as $25.13 \mathrm{G} \Omega$. The effective capacitance of the array of tunnel junctions was found to be $0.499 \mathrm{aF}$, giving a charging energy of $160.6 \mathrm{meV}$. (c) 2007 American Institute of Physics. [DOI: 10.1063/1.2761837]
\end{abstract}

\section{INTRODUCTION}

Single electron transistors (SETs), operating on the principle of Coulomb blockade (CB) in nanostructures, are promising candidates for future ultralow power and high density integrated devices. ${ }^{1-5}$ SETs can be potentially used as electron pumps ${ }^{6,7}$ for the transfer of one electron at a time and as electrometers. ${ }^{8}$ Despite the observation of single electron charging effects a quarter century ago ${ }^{9}$ the progress toward SETs operating at room temperature has been rather slow. ${ }^{10,11}$ For room temperature operation, which is a critical requirement for practical applications, the capacitance of the SET device needs to be of the order of attofarads, thus limiting the dimension of the quantum confining islands below $10 \mathrm{~nm}$. Fabrication of uniform-sized, sub- $10 \mathrm{~nm}$ quantum confining islands in a device configuration has been a major challenge in the progress of SET technology. Different methods, such as the oxidation, anisotropic wet etching, scanning tunneling microscope nano-oxidation and, side gate on thin silicon-on-insulator (SOI) substrates, have been used in the past to fabricate low-dimension quantum islands in SET devices. ${ }^{12}$ Here, we present an approach to fabricate SETs using tungsten islands that exhibit excellent room temperature operation. The key to our approach has been the application of the focused ion beam (FIB) deposition technique to create nearly uniform size nanoislands. Our fabrication method has allowed us to deposit nanoislands with a diameter of $\sim 8 \mathrm{~nm}$. Although the size of the deposited islands varied substantially in the present work, FIB technology shows the potential for better control of the deposited island size. The large value of the tunnel resistance coupled with the small island size assures room temperature operation of the device. ${ }^{13}$ The $I-V$ characteristics of the device show the $\mathrm{CB}$ and Coulomb oscillations (COs) at room temperature.

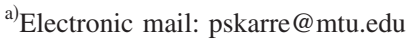

\section{EXPERIMENT}

The devices were fabricated on a Si substrate of dimensions $0.5 \times 0.5 \mathrm{~cm}^{2}$. The substrates were cleaned in a solution of $\mathrm{H}_{2} \mathrm{O}_{2}$ and $\mathrm{H}_{2} \mathrm{SO}_{4}$ in a $1: 1$ ratio (piranha solution) to remove any surface contaminants. The samples were rinsed in de-ionized water and dried in a nitrogen ambient. Subsequently, a thin film of $\mathrm{Al}_{2} \mathrm{O}_{3}$ was deposited on the silicon wafer in a Perkin-Elmer 2400-8J parallel plate rf sputtering system. This oxide layer isolates the device from the substrate; the thickness of the $\mathrm{Al}_{2} \mathrm{O}_{3}$ was $300 \mathrm{~nm}$. The silicon wafer having $\mathrm{Al}_{2} \mathrm{O}_{3}$ thin film was then introduced into the Hitachi 2000A FIB system. The Hitachi 2000A FIB system has a selection of beams with different apertures. ${ }^{14}$ Among the beams available, the beam MI-200 has a beam aperture of $200 \mu \mathrm{m}$; the beam current for MI-200 is $2.0-3.5 \mathrm{nA}$. Using the MI-200 beam, tungsten nanoparticles with an average diameter of $8 \mathrm{~nm}$ were deposited on the $\mathrm{Al}_{2} \mathrm{O}_{3}$ thin film on an area of $16 \times 16 \mu \mathrm{m}^{2}$. The tungsten nanoparticles deposited by the FIB system are shown in the scanning electron microscopy (SEM) micrograph in Fig. 1. The nanoparticles are deposited in a random arrangement over the 16 $\times 16 \mu \mathrm{m}^{2}$ area due to the competition between deposition and sputter etch. Efforts to realize single dot systems using FIB deposition are in progress. Single dot SET structures were realized in Ni using FIB etching. ${ }^{15}$ The minimum island size by FIB etching was limited to $45 \mathrm{~nm}$ and hence could not result in room temperature operation. The sample with the deposited tungsten nanoparticles was placed in piranha solution for the duration of $30 \mathrm{~s}$ to further reduce the size of the deposited nanoislands and their capacitance. The tungsten nanoparticles were then oxidized in peracetic acid, which is a combination of $\mathrm{H}_{2} \mathrm{O}_{2}$ and acetic acid, in a volume ratio of 1:1 for $4 \mathrm{~min}$. Peracetic acid, being a strong oxidizing agent, oxidizes the surface of the tungsten nanoparticles, forming a thin layer of tungsten oxide. The tunnel junctions 


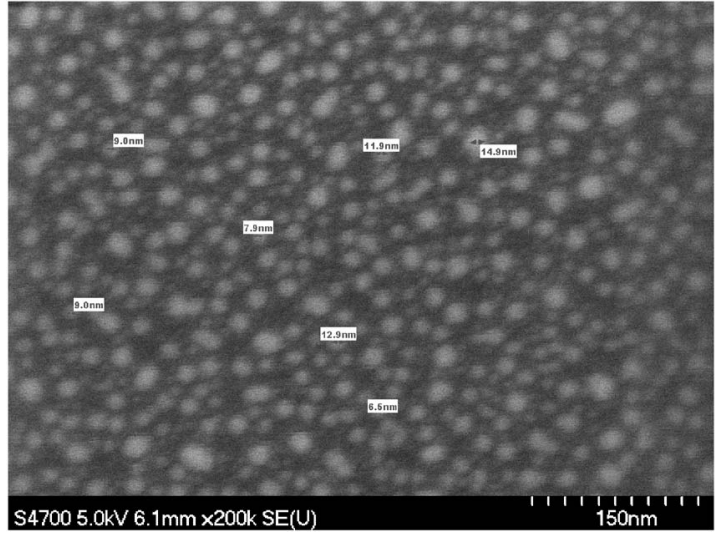

FIG. 1. Tungsten nanoparticles fabricated by FIB deposition. The average particle diameter is $8 \mathrm{~nm}$.

for the SET were fabricated during the chemical oxidation of tungsten. After a thorough clean in de-ionized water, the samples were introduced into the Hitachi 2000A FIB system to fabricate the connecting nanoleads and the microscale probing pads for the device. These electrodes and pads shown in Fig. 2 can also be fabricated by microfabrication techniques capable of $50 \mathrm{~nm}$ features. The dimensions of the probing pads for the device are $100 \times 100 \mu \mathrm{m}^{2}$ and the nanoscale connecting leads from source, drain, and gate terminals are $50 \mu \mathrm{m}$ in length and have a width of $250 \mathrm{~nm}$. The protruding structures on the source and drain nanoscale leads have dimensions of $1 \mu \mathrm{m}$ in length and $250 \mathrm{~nm}$ in width. The nanoscale tunnel leads and the probing pads for the device were also fabricated by FIB deposition, where a beam with an aperture of $50 \mu \mathrm{m}$ was used. After the deposition of the pads and the nanoscaled leads, a thin film of $\mathrm{Al}_{2} \mathrm{O}_{3}$ having a thickness of $30 \mathrm{~nm}$ was sputtered on the sample. This oxide film of $30 \mathrm{~nm}$ acts as a passivating layer for the device. A SEM micrograph of the fabricated SET structure is shown in Fig. 3. The inset of Fig. 3 shows the active area after the oxidation of the nanoparticles. The fabricated device consists of an array of tunnel junctions between the leads. From the measured average size and spacing between the nanoislands, we estimate that approximately 20 tunnel junctions per device are formed. The active area in this SEM micrograph was

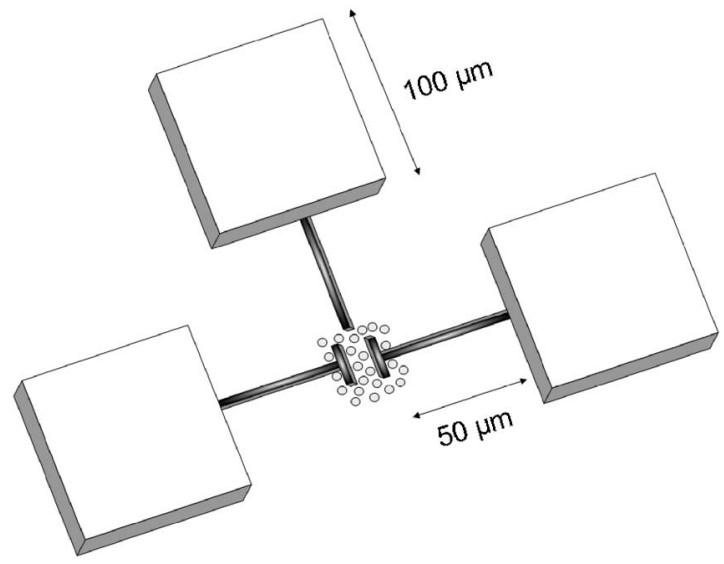

FIG. 2. Top view of the device architecture. The source and drain terminals have protruding structures near the quantum dot islands.

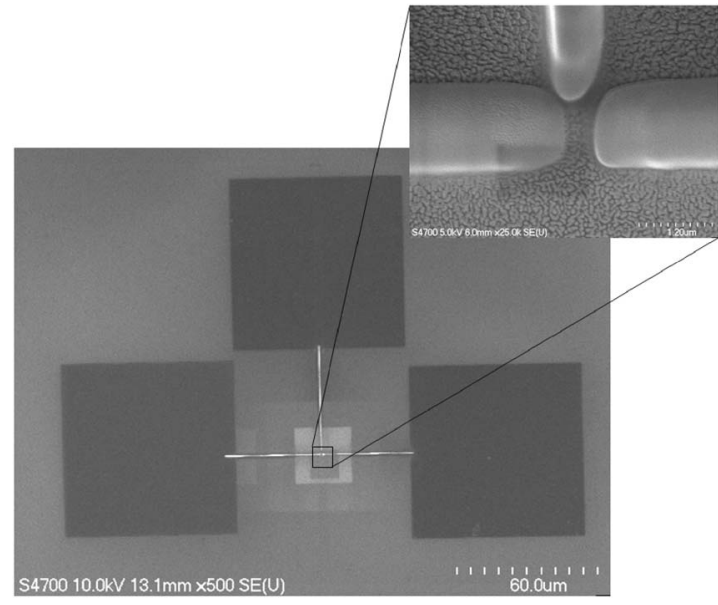

FIG. 3. SEM micrograph of the FIB deposited SET structure prior to the sputter deposition of the passivating layer. The inset in the figure shows the active area of the device; the measured device has protruding structures near the source-drain terminals.

imaged prior to the sputter deposition of the passivating layer. The device tested incorporates broad electrodes with a length of $1 \mu \mathrm{m}$ and a width of $250 \mathrm{~nm}$ at the source and drain terminals, as shown in Fig. 2. After the deposition of the blanket passivating layer of $\mathrm{Al}_{2} \mathrm{O}_{3}$, the sample was introduced in the Hitachi 2000A FIB system to expose the probing pads under the $\mathrm{Al}_{2} \mathrm{O}_{3}$ thin film. The $\mathrm{Al}_{2} \mathrm{O}_{3}$ oxide above the probing pads was etched using the beam with a current of 4.0-8.0 nA. The etch time for each pad was $10 \mathrm{~min}$ for the oxide of $30 \mathrm{~nm}$ thickness. The $I-V$ characteristics of the fabricated SET devices were measured in a custom-built vacuum probe chamber interfaced with a Keithley 4200-SCS system. The $I-V$ measurements were performed at room temperature (298 and $293 \mathrm{~K})$.

\section{RESULTS AND DISCUSSION}

The room temperature current-voltage $(I-V)$ characteristics of the device with the source-drain voltage $\left(V_{\mathrm{sd}}\right)$ swept from -3.0 to $+3.0 \mathrm{~V}$ at the gate bias $\left(V_{\mathrm{g}}\right)$ of $-3.0 \mathrm{~V}$ and $33.3 \mathrm{mV}$ are shown in Fig. 4. It can be seen from the nonlinear $I_{\mathrm{d}}-V_{\text {sd }}$ characteristics of the device in Fig. 4 that the Coulomb blockade is clearly visible for the gate voltage bias

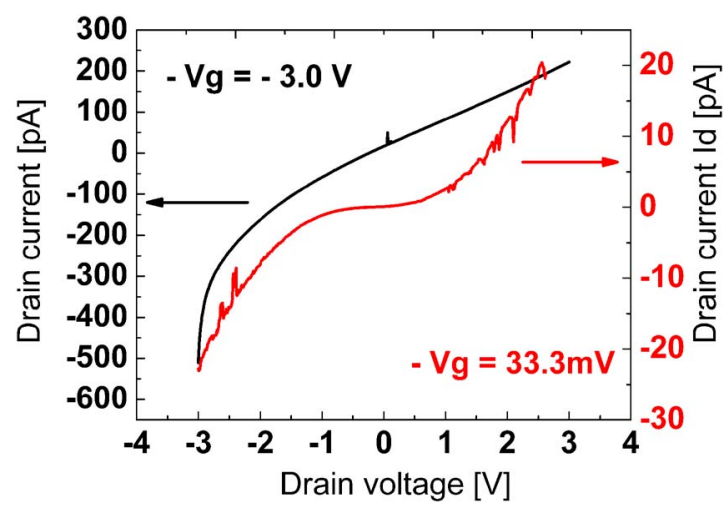

FIG. 4. (Color online) Source-drain characteristics of SET device at room temperature. Coulomb blockade is clearly visible for a lower gate bias of $33.3 \mathrm{mV}$. 


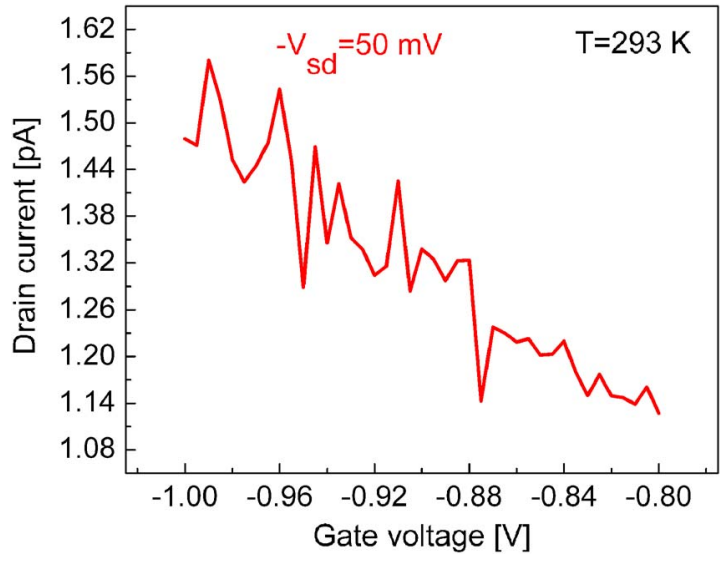

FIG. 5. (Color online) Periodic Coulomb oscillations measured at room temperature for a source-drain voltage of $50.0 \mathrm{mV}$.

of $33.3 \mathrm{mV}$. For a higher gate voltage of $-3.0 \mathrm{~V}$, the Coulomb blockade is not prominent. The drain current of the device is on the order of picoamperes, and the magnitude of the drain current changes by two orders of magnitude for a change of $3.3 \mathrm{~V}$ in gate bias.

Figure 5 shows the Coulomb oscillations in the device with respect to the gate voltage $V_{\mathrm{g}}$. The $V_{\mathrm{g}}$ was swept from -1.0 to $-0.8 \mathrm{~V}$ for a fixed $V_{\mathrm{sd}}=50.0 \mathrm{mV}$. The Coulomb oscillations are visible for the applied source-drain voltage; the drain current, which is in the picoampere range, oscillates periodically with the increase in the gate bias, showing clearly that the oscillations arise from the confinement of electrons on the tungsten quantum islands. Figure 6 shows the conductance oscillations for $V_{\mathrm{g}}$ swept from -1.0 to $-0.4 \mathrm{~V}$ for a $V_{\mathrm{sd}}$ of $50.0 \mathrm{mV}$. It can be seen that the conductance of the SET oscillates periodically with the gate voltage, confirming the Coulomb oscillations at room temperature.

For a multiple-junction device, the device parameters can be calculated from the measured data using "orthodox theory." $"$ Utilizing the measured average size of the nanoislands $(\sim 8 \mathrm{~nm})$ and the spacing between source and drain terminals, we estimate that the device consists of an array of 20 tunnel junctions. Assuming that the tunnel junctions

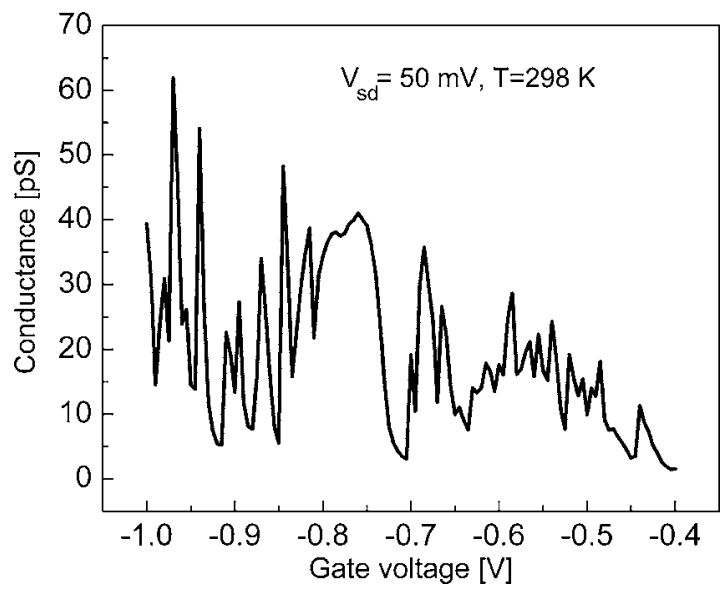

FIG. 6. Room temperature conductance oscillations of SET for a sourcedrain bias of $50.0 \mathrm{mV}$. within the SET device have the same resistance and capacitance, we can calculate the charging energy of the device according to ${ }^{17}$

$$
\Delta G / G_{T}=\left(E_{c} / 3 k_{B} T\right)[(N-1) / N],
$$

where $E_{c}$ is the charging energy $E_{c}=e^{2} / 2 C^{*}, C^{*}$ is the capacitance of the array, $N$ is the number of junctions in the array, $\Delta G$ is the dip in the measured conductance around zero source-drain bias of the device, and $G_{T}$ is the asymptotic conductance at large positive and negative source-drain biases of the device measured. The capacitance per junction $C$ is related to the total capacitance $C^{*}$ as ${ }^{18} C^{*}=N C /[2(N$ $-1)]$. The dip in the normalized conductance as measured from the conductance of the device is 0.978 , which gives a charging energy of the device, $E_{c}=160.0 \mathrm{meV}$. This gives an effective capacitance, $C^{*}$, of the device $=0.499 \mathrm{aF}$, and the capacitance of the individual junction, $C=0.947 \mathrm{aF}$. The value of the measured $E_{c}(=160 \mathrm{meV})$ is six times higher than the room temperature thermal energy, which allows the observation of the Coulomb oscillations at room temperature, as reported here. The resistance of the tunnel junctions, which is related to the charging energy, the temperature of operation, and the number of tunnel junctions in an array, ${ }^{19}$ is calculated to be $25.13 \mathrm{G} \Omega$ per tunnel junction.

\section{CONCLUSIONS}

An approach based on FIB deposition technique was developed to fabricate SETs operating at room temperature. The SETs were fabricated with tungsten islands having an average diameter of less than $8 \mathrm{~nm}$. The connecting leads and the measuring pads for the device were also fabricated with tungsten using FIB deposition technique. The fabricated SET devices clearly show Coulomb blockade and Coulomb oscillations at room temperature. The SET device consists an array of tunnel junctions. The resistance per tunnel junction of the device is calculated to be $25.13 \mathrm{G} \Omega$, which is over five orders of magnitude higher than the quantum resistance $(26.0 \mathrm{k} \Omega)$, thus enabling the confinement of electrons to the tungsten quantum dots. The effective capacitance of the device is estimated to be $0.499 \mathrm{aF}$, which gives the capacitance of individual tunnel junction $\sim 0.947 \mathrm{aF}$ and a charging energy of the device $\sim 160.6 \mathrm{meV}$. The estimated $E_{c}$ is much higher than the thermal fluctuations at room temperature, resulting in the observation of Coulomb oscillations even at room temperature.

\section{ACKNOWLEDGMENTS}

The research reported in this document was performed in connection with Contract No. DAAD17-03-C-0115 with the U.S. Army Research Laboratory. The work at ARL was partially supported by the Director's Research Initiative (Award No. 05-WMR-01).

${ }^{1}$ H. Grabert and M. H. Devaret, Single Charge Tunneling, Coulomb Blockade Phenomena in Nanostructures (Plenum, New York, 1992).

${ }^{2}$ A. Nakajima, T. Futatsugi, K. Kosemura, T. Fukano, and N. Yokoyama, Appl. Phys. Lett. 71, 353 (1997).

${ }^{3}$ T. Sakamoto, H. Kawaura, and T. Baba, Appl. Phys. Lett. 72, 795 (1998). ${ }^{4}$ R. Augke, W. Eberhardt, C. Single, F. E. Prins, D. A. Wharam, and D. P. 
Kern, Appl. Phys. Lett. 76, 2065 (2000).

${ }^{5}$ A. Tilke, R. H. Blick, and H. Lorenz, J. Appl. Phys. 90, 942 (2001).

${ }^{6}$ H. Pothier, P. Lafarge, P. F. Orfila, C. Urbina, D. Esteve, and M. H. Devoret, Physica B 169, 573 (1991).

${ }^{7}$ M. W. Keller, A. L. Eichenberger, J. M. Martinis, and N. M. Zimmerman, Science 285, 1706 (1999).

${ }^{8}$ M. H. Devoret and R. J. Schoelkopf, Nature (London) 406, 1039 (2000).

${ }^{9}$ T. A. Fulton and G. J. Dolan, Phys. Rev. Lett. 59, 109 (1987).

${ }^{10}$ K. K. Likharev, Proc. IEEE 87, 606 (1999).

${ }^{11}$ Y. Takahashi, Y. Ono, A. Fujiwara, and H. Inokawa, J. Phys.: Condens. Matter 14, R995 (2002).

${ }^{12}$ L. Zhuang, L. Guo, and S. Y. Chou, Appl. Phys. Lett. 72, 1205 (1998).

${ }^{13}$ T. J. Walls, V. A. Sverdlov, and K. K. Likharev, Physica E (Amsterdam)
19, 23 (2003).

${ }^{14}$ Hitachi FB-2000A FIB manual.

${ }^{15}$ P. S. K. Karre and P. L. Bergstrom, Proceeding of the IWPSD'05:13 International Workshop on the Physics of Semiconductor Devices, New Delhi, India, 13-17 December 2005, Vol. II, pp. 1637-1641.

${ }^{16}$ J. P. Pekola, K. P. Hirvi, J. P. Kauppinen, and M. A. Palanen, Phys. Rev. Lett. 73, 2903 (1994).

${ }^{17}$ K. P. Hirvi, J. P. Kauppinen, A. N. Korotkov, M. A. Palanen, and J. P. Pekola, Appl. Phys. Lett. 67, 2096 (1995).

${ }^{18}$ F. Giazotto, T. T. Heikkilä, A. Luukanen, A. M. Savin, and J. P. Pekola, Rev. Mod. Phys. 78, 217 (2006)

${ }^{19}$ Sh. Farhangfar, R. S. Poikolainen, J. P. Pekola, D. S. Golubev, and A. D. Zaikin, Phys. Rev. B 63, 075309 (2001). 